\title{
Beyond immunosuppression: reconsidering indoleamine 2,3-dioxygenase as a pathogenic element of chronic inflammation
}

\author{
"...elevated tryptophan degradation has been correlated with disease activity, \\ suggesting the possibility that [indoleamine 2,3-dioxygenase] might simlarly play \\ a role in inflammatory autoimmune pathologies."
}

This article highlights recent findings that have led us to re-evaluate the role of indoleamine 2,3-dioxygenase (IDO) as a conventional immunosuppressive actor. In the context of de novo carcinogenesis, we found that IDO was induced by the inflammatory tumor-promoting process itself, independent of tumor immunoediting. Furthermore, loss of IDO did not exacerbate the severity of inflammation as might be expected if it were broadly immunosuppressive. Rather, IDO appears to be integrally involved in shaping the inflammatory environment to support tumor outgrowth. Intriguingly, in patients with rheumatoid arthritis or systemic lupus erythematosus, elevated tryptophan degradation has been correlated with disease activity, suggesting the possibility that IDO might similarly play a role in inflammatory autoimmune pathologies. Consistent with this idea, in a spontaneous mouse model of arthritis, we found that IDO is elevated at the onset of disease, while treatment with the IDO inhibitor 1-methyl tryptophan (1MT) significantly reduced disease severity. These findings add to the growing body of evidence suggesting that IDO is a key element shaping the pathogenic nature of the chronic inflammatory environment. NF- $\mathrm{KB}$ signaling plays a central role in both inflammation and cancer, leading us to speculate that it might act as a common integrator of IDO activity in these complex biological processes. We probed this connection using ethyl pyruvate (EP), a small molecule that directly interferes with canonical $\mathrm{NF}-\kappa \mathrm{B}$ signaling, demonstrating that EP can induce robust antitumor immune responses in preclinical models through its ability to inhibit IDO induction in vivo. These studies reveal a possible role for $\mathrm{NF}-\kappa \mathrm{B}$ in supporting cancer development through IDO-dependent immune escape and indicate that IDO may be a general determinant for shaping the pathogenic character of chronic inflammation.

\section{"...[indoleamine 2,3-dioxygenase] is a key element shaping the pathogenic nature of the chronic inflammatory environment."}

Immune escape in cancer, which manifests as dominant suppression of antitumor immunity, has become widely recognized as one of the major impediments to effective immunotherapy. A number of different actors implicated in immune escape may be particularly attractive as potential targets for the development of small molecule therapeutics [1]. One key actor in this process is the enzyme IDO. Recently, it has been found that independent genes encode two related IDO isoforms, and it remains to be determined whether the newly identified IDO2encoded isoform $[2,3]$ has distinct physiological relevance that is separate from the extensively studied isoform encoded by IDOI. IDO catalyzes the initial and rate-limiting step in the degradation of tryptophan along a pathway that can lead to the biosynthesis of $\mathrm{NAD}^{+}[4]$. However, IDO does not handle dietary catabolism of tryptophan, which is instead the role of the structurally unrelated liver-specific enzyme tryptophan dioxygenase, nor does it appear to be critical for maintaining NAD ${ }^{+}$levels, which, in mammalian cells, is predominantly the purview of salvage pathways. Instead, IDO1 is an IFN- $\gamma$ inducible gene and its pattern of expression suggested early on an involvement in inflammatory processes [5]. The striking demonstration that administration of the specific bioactive IDO inhibitor 1MT [6] could elicit MHC-restricted, T-cell-mediated rejection of allogeneic mouse concepti $[7,8]$ firmly established IDO as a physiologically relevant immunomodulatory enzyme. This, coupled with data that IDO could suppress activation of effecter $T$ cells [9], led to the concept of IDO as an immunosuppressive actor involved in the establishment of acquired peripheral immune tolerance. However, the idea that

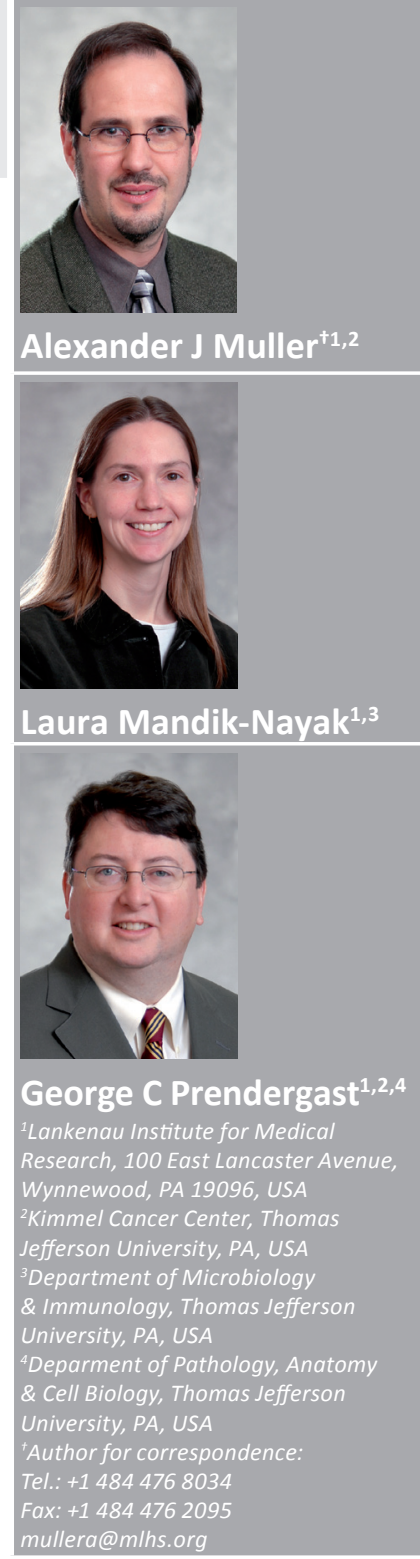

future medicine $^{\text {pot }}$ fsg 
IDO activity is simply immunosuppressive may need to be re-evaluated based on emerging evidence suggesting a more complex and nuanced role for IDO in the development of pathologies associated with chronic inflammation.

Recent studies based on the classical two-stage 7,12-dimethylbenz $[a]$ anthracene (DMBA)/ phorbol 12-myristate 13-acetate (TPA; or PMA) skin carcinogenesis protocol have provided direct genetic validation of the theory that IDO has an important effector role in the process of de novo tumorigenesis [10]. In this model, tumors are initiated with a single application of the carcinogen DMBA. This step is followed by multiple rounds of exposure to the proinflammatory phorbol ester TPA, which elicits a state of chronic inflammation in the skin that promotes tumor outgrowth. In this model, it was demonstrated that mice in which both alleles of the Ido1 gene were genetically disrupted (Ido1-nullizygous) are quite resistant to the formation of premalignant papillomas, as well as to their subsequent conversion to malignant carcinomas [10].

"...the idea that IDO [indoleamine
2,3-dioxygenase] activity is simply
immunosuppressive may need to be
re-evaluated based on emerging evidence
suggesting a more complex and nuanced role
for IDO in the development of pathologies
associated with chronic inflammation."

One question raised by these findings is, at what stage in the process of tumorigenesis is IDO relevant? The multistage aspect of the DMBA/TPA carcinogenesis protocol provided us with a unique opportunity to investigate this question in the context of de novo tumor development. Immunoediting has been proposed as a conceptual framework within which to understand how a tumor develops in the context of the host's immune system. The immunoediting process has been broken down into the three phases of elimination, equilibrium and escape [11], and it has generally been assumed that elevated IDO activity is a relatively late event that is driven by selective pressure on the tumor to escape elimination. This interpretation would require there to be at least some nascent tumor present for IDO to be induced. Instead, however, we found that TPA treatment alone was sufficient to induce IDO in the proximal lymph nodes [10]. Because these mice were never exposed to DMBA initiation, this elevation of IDO occurred in the absence of cancer, as TPA alone is not able to drive the development of neoplasia in the absence of an initiating agent. Therefore, this outcome is more in line with the elevation of IDO being an early event driven by inflammation rather that a late event driven by immune selection.

Based on the generally accepted characterization of IDO as an immunosuppressive enzyme, inflammation might be expected to run rampant in an Ido1-nullizygous mouse in which this immunosuppressive check is no longer in place. However, when Ido1-nullizygous mice treated with TPA were compared with wild-type control animals receiving the same treatment, the severity of the inflammation that developed was not found to be discernibly different [Muller AJ ET AL., Unpublished Observations]. So, rather than IDO simply being an immunosuppressive counterbalance in inflammatory reactions, these data instead suggest a more nuanced interpretation whereby IDO, acting as an integral component of inflammation, shapes its pathogenic capacity to promote tumor development.

The conceptual realization that IDO may act as an integral component within the inflammatory milieu in order to shape its pathogenic capacity to support tumor development led us to consider the broader question of whether IDO might similarly contribute to the development of other pathologies associated with chronic inflammation. Indeed, evidence that elevated tryptophan degradation correlates with disease activity in both rheumatoid arthritis and systemic lupus erythematosus patients suggests that increased IDO activity may play an activating role in the autoimmune response in humans [12,13]. Direct corroborative evidence for this idea came from studies we performed in the $\mathrm{K} / \mathrm{BxN}$ spontaneous mouse model of arthritis [14]. IDO activity was elevated at the onset of disease, and treatment of these animals with the small-molecule inhibitor of IDO, $1 \mathrm{MT}$, resulted in significant alleviation of the joint inflammation that develops in this model, with 1MT-treated animals exhibiting minimal synovial expansion and fewer infiltrating inflammatory cells [15]. 1MT treatment had no apparent effect on the percentage of regulatory $\mathrm{T}$ cells or the levels of Th1/Th2/Th17 cytokines, but did greatly diminish the autoreactive $\mathrm{B}$-cell response, indicative of a novel role for IDO in contributing to the development of autoimmune disease by supporting the activation of autoreactive B cells. In conjunction with the carcinogenesis results, these data strongly argue that the role of IDO in chronic inflammation is more complex than simply acting as an 
immunosuppressive brake. Rather, IDO appears to alter inflammatory pathogenesis in these two models, exacerbating the development of tumors in the context of the DMBA/TPA carcinogenesis model and of autoimmune arthritis in the $\mathrm{K} / \mathrm{BxN}$ model.

"... although there may be other contributory effects of [ethyl pyruvate], the majority of its antitumor activity depends on its ability to suppress [indoleamine 2,3-dioxygenase]."

At the molecular level, NF- $\kappa \mathrm{B}$ transcription factors, as nodal integrators for signaling pathways driving both inflammation and cancer, may represent a shared interface between these two complex biological processes. Our previous work had suggested a potential role for canonical $\mathrm{NF}-\kappa \mathrm{B}$ signaling in controlling the induction of Ido1 promoter activity that was comparable in magnitude to the well-validated requirement for JAK/STAT signaling [16]. Following-up on this evidence, we have explored the use NF- $\mathrm{KB}$ inhibitory compounds to further probe the connection between NF- $\kappa \mathrm{B}$ signaling and IDO regulation. Pioneering studies from Fink's group have demonstrated that the simple, nontoxic compound EP can interfere with NF- $\kappa \mathrm{B}$ nuclear translocation and DNA binding through a mechanism that involves covalent interaction with $\mathrm{Cys}^{38}$ of the p65 (RelA) subunit [17,18], an essential component of canonical NF- $\kappa \mathrm{B}$ signaling. Importantly, $\mathrm{EP}$ has demonstrated in vivo biological activity associated with NF- $\kappa \mathrm{B}$ inhibitory action in several preclinical models of inflammation-related disorders [19-22]. As anticipated from the reporter assay data, EP was able to effectively inhibit IDO induction both in vitro and in vivo, at concentrations where it had been found to interfere with NF- $\kappa$ B activity [23]. Previously, we had demonstrated that direct inhibitors of IDO enzymatic activity are effective at suppressing the outgrowth of tumors formed by engrafted B16-F10 melanoma cells [24-26]. Owing to the fact that these tumors are both highly malignant and poorly immunogenic, they provided a stringent system in which to evaluate the biological activity of EP. Consistent with the ability of EP to block IDO, the outgrowth of established B16-F10 tumors was significantly suppressed by EP treatment [23]. However, there could be any number of possible explanations for why a NF- $\kappa \mathrm{B}$ inhibitor might suppress tumor outgrowth. One of the distinguishing characteristics of IDO inhibitors in this tumor model is their absolute dependence on intact T-cell tumor immunity for their antitumor effect. Furthermore, because the tumors formed by the B16-F10 melanoma line do not express IDO within the tumor cells themselves, their dependence on IDO is completely based on extrinsic expression of the enzyme in normal host cells. As predicted for an inhibitor of IDO, the antitumor activity of EP was abrogated in athymic, T-cell-deficient mice, demonstrating that it is not directly impacting the growth characteristics of the tumor cells within the animal [23]. EP efficacy was also severely compromised in Ido1nullizygous animals, indicating that although there may be other contributory effects of EP, the majority of its antitumor activity depends on its ability to suppress IDO [23].

$\mathrm{NF}-\kappa \mathrm{B}$ signaling can contribute to tumorigenesis in a variety ways, including having a direct impact on tumor development by driving proliferation, survival and metastatic capability, as well as by shaping the external microenvironment as a driver of inflammation and angiogenesis [27]. Given the multifactoral involvement of $\mathrm{NF}-\kappa \mathrm{B}$ as a signaling node in cancer development, it was somewhat surprising how dominant the IDO-mediated immune escape contribution appeared to be in the context of EP treatment. This may be in part related to the choice of tumor graft model, as B16-F10 melanomas are illustrative of a class of tumors that preferentially use host-cell expressed IDO as an immune escape mechanism, so that once a tumor has become established, continued IDO activity must be sustained in order to maintain the immunoprivileged state. We have used the term 'tolerance addiction' to describe this process in order to highlight the underlying congruity with the idea in cancer genetics of 'oncogene addiction', in which cancer cells exhibit physiological dependence on the continued activity of an initiating oncogenic event in order to maintain their malignant phenotype [28]. Conceptually, tolerance addiction explains why acute interference with IDO activity, as with a pharmacologic agent, causes immunogenic unmasking that contributes to tumor rejection. However, if upregulation of IDO activity is not possible from the outset (as in an Ido1-nullizygous animal), it appears that alternative immune escape mechanisms can be accessed by the developing tumor, in which case IDO-targeting compounds are rendered ineffectual. Interestingly, this ability to access compensatory immune escape mechanisms in the absence of IDO appears to be recapitulated in the context of fetal development as well [29]. Although further work will be necessary in 
order to determine the broader relevance of this immunoregulatory aspect of $\mathrm{NF}-\kappa \mathrm{B}$ in other tumor systems, these results suggest that IDOmediated immune escape should be considered as another fundamental contribution of $\mathrm{NF}-\kappa \mathrm{B}$ signaling to tumorigenesis and that the role of $\mathrm{NF}-\kappa \mathrm{B}$ in regulating IDO induction may be important in the pathobiology of other diseases associated with chronic inflammation.

\section{Acknowledgements}

We thank Dr Richard Metz and Dr Lisa Laury-Kleintop for helpful discussions.

\section{Financial \& competing interests disclosure} This work is supported by the Lankenau Hospital Foundation (to Alexander J Muller, Laura MandikNayak and George C Prendergast). Alexander J Muller is the recipient of a Lance Armstrong Foundation Grant. George C Prendergast is the recipient of NIH Grants CA109542, CA82222 and CA100123. Alexander J Muller, Laura Mandik-Nayak and George $C$ Prendergast declare intellectual property interests related to indoleamine 2,3-dioxygenase (IDO). Alexander J Muller and George C Prendergast also declare financial interests, grant support and consultancy roles with New Link Genetics Corporation, which is engaged in the clinical development of IDO inhibitors for the purpose of treating cancer and other diseases. The authors have no other relevant affiliations or financial involvement with any organization or entity with a financial interest in or financial conflict with the subject matter or materials discussed in the manuscript apart from those disclosed.

No writing assistance was utilized in the production of this manuscript.

\section{Bibliography}

1 Muller AJ, Scherle PA: Targeting the mechanisms of tumoral immune tolerance with small-molecule inhibitors. Nat. Rev. Cancer 6(8), 613-625 (2006).

2 Ball HJ, Sanchez-Perez A, Weiser S et al.: Characterization of an indoleamine 2,3-dioxygenase-like protein found in humans and mice. Gene 396(1), 203-213 (2007).

3 Metz R, Duhadaway JB, Kamasani U, Laury-Kleintop L, Muller AJ, Prendergast GC: Novel tryptophan catabolic enzyme IDO2 is the preferred biochemical target of the antitumor indoleamine 2,3-dioxygenase inhibitory compound D-1-methyl-tryptophan. Cancer Res. 67(15), 7082-7087 (2007).

4 Shimizu T, Nomiyama S, Hirata F, Hayaishi O: Indoleamine 2,3-dioxygenase. Purification and some properties. J. Biol. Chem. 253(13), 4700-4706 (1978).

5 Yoshida R, Hayaishi O: Induction of pulmonary indoleamine 2,3-dioxygenase by intraperitoneal injection of bacterial lipopolysaccharide. Proc. Natl Acad. Sci. USA 75(8), 3998-4000 (1978).

6 Cady SG, Sono M: 1-methyl-DL-tryptophan, $\beta$-(3-benzofuranyl)-DL-alanine (the oxygen analog of tryptophan), and $\beta$-[3-benzo(b) thienyl]-DL-alanine (the sulfur analog of tryptophan) are competitive inhibitors for indoleamine 2,3-dioxygenase. Arch. Biochem. Biophys. 291, 326-333 (1991).

7 Mellor AL, Sivakumar J, Chandler P et al.: Prevention of T cell-driven complement activation and inflammation by tryptophan catabolism during pregnancy. Nat. Immunol. 2(1), 64-68 (2001).
8 Munn DH, Zhou M, Attwood JT et al.: Prevention of allogeneic fetal rejection by tryptophan catabolism. Science 281, 1191-1193 (1998).

9 Munn DH, Shafizadeh E, Attwood JT, Bondarev I, Pashine A, Mellor AL: Inhibition of $\mathrm{T}$ cell proliferation by macrophage tryptophan catabolism. J. Exp. Med. 189, 1363-1372 (1999).

10 Muller AJ, Sharma MD, Chandler PR et al.: Chronic inflammation that facilitates tumor progression creates local immune suppression by inducing indoleamine 2,3 dioxygenase. Proc. Natl Acad. Sci. USA 105(44), 17073-17078 (2008).

11 Dunn GP, Old LJ, Schreiber RD: The immunobiology of cancer immunosurveillance and immunoediting. Immunity 21(2), 137-148 (2004).

12 Pertovaara M, Hasan T, Raitala A et al.: Indoleamine 2,3-dioxygenase activity is increased in patients with systemic lupus erythematosus and predicts disease activation in the sunny season. Clin. Exp. Immunol. 150 (2), 274-278 (2007).

13 Schroecksnadel K, Winkler C, Duftner C, Wirleitner B, Schirmer M, Fuchs D: Tryptophan degradation increases with stage in patients with rheumatoid arthritis. Clin. Rheumatol. 25(3), 334-337 (2006).

14 Mandik-Nayak L, Allen PM: Initiation of an autoimmune response: insights from a transgenic model of rheumatoid arthritis. Immunol. Res. 32(1-3), 5-13 (2005).

15 Scott GN, DuHadaway J, Pigott E et al.: The immunoregulatory enzyme IDO paradoxically drives B cell-mediated autoimmunity. J. Immunol. 182(12), 7509-7517 (2009).
16 Muller AJ, Duhadaway JB, Donover PS, Sutanto-Ward E, Prendergast GC: Inhibition of indoleamine 2,3-dioxygenase, an immunoregulatory target of the cancer suppression gene Binl, potentiates cancer chemotherapy. Nat. Med. 11(3), 312-319 (2005).

17 Han Y, Englert JA, Yang R, Delude RL, Fink MP: Ethyl pyruvate inhibits nuclear factor- $\mathrm{KB}$-dependent signaling by directly targeting p65. J. Pharmacol. Exp. Ther. 312(3), 1097-1105 (2005).

18 Sappington PL, Cruz RJ Jr, Harada T et al.: The ethyl pyruvate analogues, diethyl oxaloproprionate, 2-acetamidoacrylate, and methyl-2-acetamidoacrylate, exhibit antiinflammatory properties in vivo and/or in vitro. Biochem. Pharmacol. 70(11), 1579-1592 (2005).

19 Uchiyama T, Delude RL, Fink MP: Dose-dependent effects of ethyl pyruvate in mice subjected to mesenteric ischemia and reperfusion. Intensive Care Med. 29(11), 2050-2058 (2003).

20 Yang R, Gallo DJ, Baust JJ et al.: Ethyl pyruvate modulates inflammatory gene expression in mice subjected to hemorrhagic shock. Am. J. Physiol. Gastrointest. Liver Physiol. 283(1), G212-221 (2002).

21 Yang R, Han X, Delude RL, Fink MP: Ethyl pyruvate ameliorates acute alcoholinduced liver injury and inflammation in mice. J. Lab. Clin. Med. 142(5), 322-331 (2003).

22 Yang R, Uchiyama T, Alber SM et al:: Ethyl pyruvate ameliorates distant organ injury in a murine model of acute necrotizing pancreatitis. Crit. Care Med. 32(7), 1453-1459 (2004). 
23 Muller AJ, DuHadaway JB, Jaller D, Curtis P, Metz R, Prendergast GC: Immunotherapeutic suppression of indoleamine 2,3-dioxygenase and tumor growth with ethyl pyruvate. Cancer Res. 70(5), 1845-1853 (2010).

24 Banerjee T, DuHadaway JB, Gaspari P et al: A key in vivo antitumor mechanism of action of natural product-based brassinins is inhibition of indoleamine 2,3-dioxygenase. Oncogene 27(20), 2851-2857 (2008).
25 Gaspari P, Banerjee T, Malachowski WP et al.: Structure-activity study of brassinin derivatives as indoleamine 2,3-dioxygenase inhibitors. J. Med. Chem. 49(2), 684-692 (2006).

26 Kumar S, Malachowski WP, Duhadaway JB et al.: Indoleamine 2,3-dioxygenase is the anticancer target for a novel series of potent naphthoquinone-based inhibitors. J. Med. Chem. 51(6), 1706-1718 (2008).

27 Baud V, Karin M: Is NF- $\kappa$ B a good target for cancer therapy? Hopes and pitfalls. Nat. Rev. Drug Discov. 8(1), 33-40 (2009).
28 Weinstein IB: Cancer. Addiction to oncogenes - the Achilles heal of cancer. Science 297(5578), 63-64 (2002).

29 Baban B, Chandler P, McCool D, Marshall B, Munn DH, Mellor AL: Indoleamine 2,3-dioxygenase expression is restricted to fetal trophoblast giant cells during murine gestation and is maternal genome specific. J. Reprod. Immunol. 61(2), 67-77 (2004) 